OPEN ACCESS

Edited by:

Yan Xiang,

The University of Texas Health Science Center at San Antonio, United States

Reviewed by: Wolf-Dieter Schubert, Shenzhen Institutes of Advanced Technology (CAS), China Fan Pan,

Shenzhen Institutes of Advanced Technology, Chinese Academy of Sciences (CAS), China

*Correspondence: Jinhui Tao

taojinhui@ustc.edu.cn

${ }^{\dagger}$ These authors have contributed equally to this work

Specialty section: This article was submitted to Structural Biology,

a section of the journal

Frontiers in Molecular Biosciences

Received: 23 February 2021 Accepted: 20 May 2021 Published: 04 June 2021

Citation: Zhang H, Tang $Y$ and Tao J (2021) Sex-Related Overactivation of NLRP3 Inflammasome Increases Lethality of the Male COVID-19 Patients. Front. Mol. Biosci. 8:671363. doi: 10.3389/fmolb.2021.671363

\section{Sex-Related Overactivation of NLRP3 Inflammasome Increases Lethality of the Male COVID-19 Patients}

\author{
Hongliang Zhang ${ }^{\dagger}$, Yujie Tang ${ }^{\dagger}$ and Jinhui Tao *
}

Department of Rheumatology and Immunology, The First Affiliated Hospital of USTC, Division of Life Sciences and Medicine, University of Science and Technology of China, Hefei, China

The COVID-19 pandemic, caused by SARS-CoV-2 infection, remains a dramatic threat to human life and economic well-being worldwide. Significant heterogeneity in the severity of disease was observed for patients infected with SARS-CoV-2 ranging from asymptomatic to severe cases. Moreover, male patients had a higher probability of suffering from high mortality and severe symptoms linked to cytokine storm and excessive inflammation. The NLRP3 inflammasome is presumably critical to this process. Sex differences may directly affect the activation of NLRP3 inflammasome, impacting the severity of observed COVID19 symptoms. To elucidate the potential mechanisms underlying sex based differences in NLRP3 activation during SARS-CoV-2 infection, this review summarizes the reported mechanisms and identifies potential therapeutic targets.

Keywords: SARS-CoV-2, COVID-19, NLRP3 inflammasome, sex, cytokine storm, P2X7R

\section{INTRODUCTION}

Coronaviridae are plus-sense single strand RNA viruses subdivided into Coronavirinae and Torovirinae. Some members of the Coronavirinae including severe acute respiratory syndrome coronavirus (SARS-CoV) and Middle East respiratory syndrome coronavirus (MERS-CoV) have drawn much attention in the past decades due to their threat to human life. At present, severe acute respiratory syndrome coronavirus 2 (SARS-CoV-2) and the resulting coronavirus disease 2019 (COVID-19) is still impacting human life around the world. Significant sex and age heterogeneity is observed between asymptomatic COVID-19 patients, compared to those with mild or severe disease. Studies have repeatedly shown that severe and fatal COVID-19 cases are most prevalent among the elderly compared to young and middle-aged patients. Most young and middle-aged patients with severe illness are male (Chen et al., 2020; Gebhard et al., 2020; Klein et al., 2020; Li et al., 2020; Meng et al., 2020). Since severe cases result in higher mortality, preventing the transition from mild to severe disease could significantly reduce mortality. In particular, identifying mechanisms linking young male COVID-19 patients to severe disease would be highly relevant.

A sudden increase of inflammation is typical for the transition of COVID-19 from mild to severe or critical disease brought about by a cytokine storm (Caricchio et al., 2021). Plasma levels of IL-1 $\beta$, $-7,-8,-9,-10$, FGF, G-CSF, GM-CSF, IFN- $\gamma$, IP-10, MCP-1, MIP-1A, MIP1-B, PDGF, TNF- $\alpha$ and VEGF are significantly elevated in severe patients, and the levels are related to the prognosis of patients (Huang C. et al., 2020). Preventing excessive cytokine production could thus help prevent progression from mild to severe disease.

IL- $1 \beta$ and its downstream effectors IL- 6 and TNF- $\alpha$ are linked to the exacerbation of COVID-19. Clinically, targeting IL-1 $\beta$, IL-6, and TNF- $\alpha$ have gained some success in severe COVID-19 patients (Xu X. et al., 2020; Dimopoulos et al., 2020; Rizk et al., 2020). These cytokines are produced in the 
earlier phase of infection by the innate immune system and are largely responsible for later induction of excessive inflammation. Preventing the secretion of these cytokines may thus help to prevent cytokine storm.

The NLRP3 inflammasome is a multiprotein complex consisting of NLRP3, ASC and procaspase-1. It recognizes both damage-associated molecular patterns (DAMPs) and pathogen-associated molecular patterns (PAMPs). After activation of the NLRP3 inflammasome, procaspase-1 is cleaved to active caspase-1, which in turn promotes the maturation and secretion of pro-inflammatory cytokines IL-1 $\beta$ and IL-18, triggering an inflammatory response (Schroder and Tschopp, 2010; Yang et al., 2019). NLRP3 inflammasome overactivation produces excessive IL-1 $\beta$ and downstream cytokines such as IL- 6 and TNF- $\alpha$, are also observed in acute respiratory distress syndrome (ARDS), ventilator induced lung injury (VILI), and disseminated intravascular coagulation (DIC). In addition, inflammatory damage of the heart, kidney, digestive and nervous systems result in COVID-19 patients (de Rivero Vaccari et al., 2020). Thus NLRP3 inflammasome overactivation may be responsible for SARS-CoV-2 induced cytokine storm in COVID-19 patients. Physiological and biochemical characteristics different between males and females may activate the NLRP3 inflammasome to varying degrees resulting in different inflammatory symptoms and prognosis. To further analyze the mechanisms underlying the cytokine storm after SARS-CoV-2 infection and the heterogeneity observed between males and females, this review summarizes the reported mechanisms of sex impact on NLRP3 inflammasome activation and proposes the potential therapeutic targets for COVID-19 treatment.

\section{ROLE OF NLRP3 INFLAMMASOME ACTIVATION IN PATIENTS WITH COVID-19}

Studies have repeatedly shown that coronavirus infection can trigger the activation of NLRP3 inflammasome through different ways. Notably, the coronavirus genome-encoded proteins contribute significantly to this activation. For example, SARS$\mathrm{CoV}$ genome encodes a variety of proteins (Kim et al., 2020), including viroporin that forms ion channels in the membrane and promotes an ionic flux to activate NLRP3 inflammasome. ORF8a, ORF3a and E proteins are all viroporins (Xu et al., 2020a). E protein forms ion channels on the ERGIC/Golgi membrane and mediates the influx of calcium ions to activate NLRP3 inflammasome (NietoTorres et al., 2015). ORF3 induces NLRP3 inflammasome activation not only in an ion channel-dependent manner (Chen et al., 2019), but also via TNF receptor-associated factor 3 (TRAF3)mediated ASC ubiquitination (Siu et al., 2019). Other proteins, such as SUD and ORF8b, can also activate NLRP3 inflammasome (Shi et al., 2019; Chang et al., 2020). Since SARS-CoV-2 shares $79.6 \%$ sequence identity with SARS-CoV (Zhou et al., 2020), it is reasonable to infer that the molecular mechanisms of NLRP3 inflammasome activation in SARS-CoV are also applicable in the case of SARS-CoV-2. Indeed, recent studies have shown that SARS-CoV-2 genome-encoded proteins, such as spike glycoprotein, can trigger the activation of NLRP3 inflammasome (Pan et al., 2020; Theobald et al., 2020).

In addition to the direct activation induced by genomeencoded proteins, SARS-CoV-2 can also activate NLRP3 inflammasome in an indirect way. For instance, it has been reported that MERS-CoV could activate NLRP3 inflammasome in a complement receptor-dependent manner (Jiang et al., 2019). The binding of SARS-CoV-2 spike glycoprotein with angiotensin-converting enzyme 2 (ACE2) triggers a series of complex molecular events, and ultimately leads to a hyper-inflammatory state. Both renin-angiotensinaldosterone system (RAAS) and complement cascade are involved in SARS-CoV-2-induced overproduction of inflammatory cytokines (Mahmudpour et al., 2020), and this overproduction is caused by NLRP3 inflammasome (Ratajczak and Kucia, 2020). Besides, the ATP released by pyroptosis-related inflammatory cells also influences the activation of NLRP3 inflammasome via ATP-P2X7R pathway.

NLRP3 inflammasome plays a major role in mediating the immune response against SRAS-CoV-2. The inflammatory factors produced by NLRP3 inflammasome could promote antigen-presenting cells to upregulate co-stimulatory molecules (such as CD40, CD80 and CD86), thus strengthening the adaptive immune responses and enhancing viral clearance. In general, moderate inflammatory response is beneficial to eliminate the virus and repair the damaged tissue. However, if NLRP3 inflammasome has a low or impaired response to the virus, it will lead to a low-level of inflammatory response, which in turn weakens the immune system and protects the virus from clearance. For example, it has been reported that the leucine-rich repeat (LRR) domain mutation of bat NLRP3 inhibits the activation of NLRP3 inflammasome during MERS-CoV infection, leading to an impairment of the immune system (Ahn et al., 2019). Therefore, it is speculated that bats may carry MERS-CoV and other coronavirus without symptoms. On the other hand, overactivation of NLRP3 inflammasome produces excessive DAMPs, causing pyroptosis, neutrophil infiltration, macrophage activation, Th17 differentiation and excessive production of inflammatory cytokines, which ultimately leads to tissue damage and fibrosis (Lin et al., 2019; van den Berg and Te Velde, 2020). For example, IL-1 $\beta$, which is induced by NLRP3 inflammasome activation to protect the host in the early phase of a viral infection, can also have serious negative consequences if the excessive production persists throughout the infection (Tate et al., 2016). Thus, it is necessary to control the activation of NLRP3 inflammasome.

Previous studies have reported that NLRP3 inflammasome and IL-1 $\beta$ are involved in lung injury and ARDS (Kolb et al., 2001; Ganter et al., 2008). The higher levels of Caspase-1 p20 and IL-18 indicated more severe symptoms and worse prognosis in patients with COVID-19 (Rodrigues et al., 2021), suggesting that NLRP3 inflammasome is not only activated but also plays a key role in COVID-19 progression. Moreover, dysregulation of NLRP3 inflammasome activity was observed in patients with severe COVID-19 (van den Berg and Te Velde, 2020). Hence, targeting NLRP3 inflammasome may be an effective strategy 


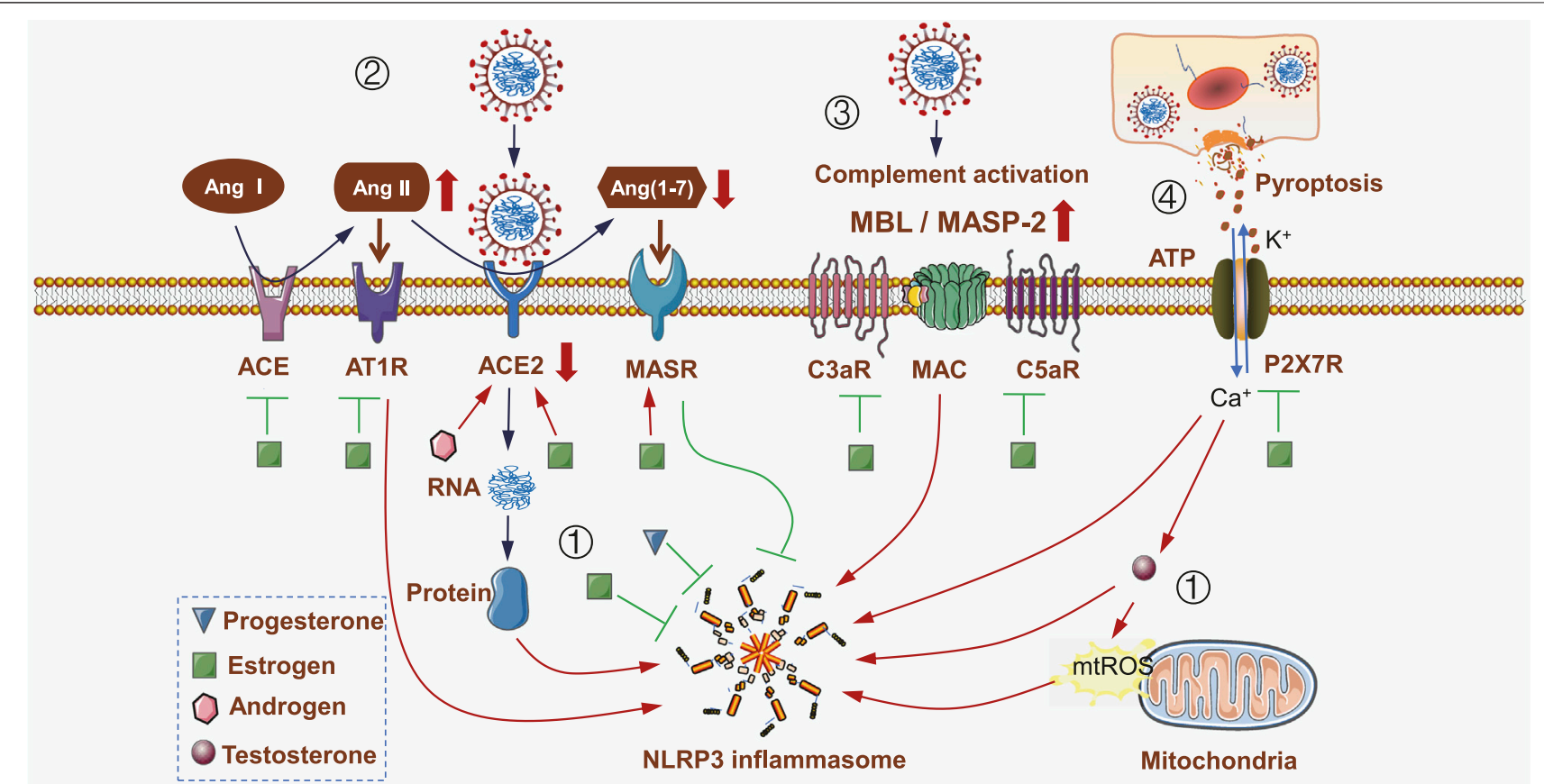

FIGURE 1 | Impact of sex differences on NLRP3 inflammasome activities during SARS-CoV-2 infection. (i) SARS-CoV-2 genome-encoded proteins directly activate NLRP3 inflammasome. Estrogen and progestin directly inhibit NLRP3 inflammasome. However, the increased level of testosterone during SARS-CoV-2 infection promotes NLRP3 inflammasome activation in a ROS-dependent manner. (ii) SARS-CoV-2 hijacks ACE2 to mediate virus invasion and replication. ACE2 is then downregulated, leading to a decrease in Ang (1-7) level, and ultimately weakens MASR-mediated inhibition of NLRP3 inflammasome; meanwhile, Ang II is upregulated and interacts with AT1R to activate NLRP3 inflammasome. Both androgen and estrogen inhibit NLRP3 inflammasome by increasing ACE2 expression. However, estrogen also inhibits ACE and ATR1, while upregulates MASR, thus leading to NLRP3 inflammasome inhibition. (iii) SARS-CoV-2 infection induces the activation of complement cascade by increasing the levels of MBL, MASP-2, C3aR, C5aR and MAC, which in turn activates NLRP3 inflammasome. Estrogen inhibits the activation of complement cascade to inhibit NLRP3 inflammasome. (iv) SARS-CoV-2 infection induces pyroptosis and releases extracellular ATP, which acts on P2X7R and results in an ionic flux to activate NLRP3 inflammasome; meanwhile testosterone is induced to activate NLRP3 inflammasome. Estrogen inhibits P2X7R, thus suppressing the activation of NLRP3 inflammasome. Triangle represents progesterone, square represents estrogen, hexagon represents androgen, and circle represents testosterone.

for the treatment of severe COVID-19 patients (Freeman and Swartz, 2020).

\section{IMPACT OF SEX DIFFERENCES ON NLRP3 INFLAMMASOME ACTIVATION INDUCED BY SARS-COV-2}

Under disease conditions, NLRP3 inflammatory components are differently expressed in a sex-dependent manner and closely associated with the development of hyper-inflammation. For example, the expression levels of NLRP3, ASC, CASP1 and IL1B in male patients with abdominal aortic aneurysm were remarkably increased compared to those in female patients, and were closed related to disease progression (Wu et al., 2016). Moreover, the activity of NLRP3 inflammasome can be regulated by biological sex. A recent study demonstrated that male COVID-19 patients had higher plasma levels of innate immune cytokines, such as IL-8 and IL-18, along with more robust induction of non-classical monocytes (Takahashi et al., 2020), suggesting that sex difference is a major factor affecting the activation of NLRP3 inflammasome. Indeed, biological sex exerts a substantial impact on NLRP3 inflammasome activation. As shown in Figure 1, different NLRP3 inflammasome activating signals were respectively regulated by sex elements in varying degrees, thus leading to different degrees of inflammation and different types of symptoms in COVID-19 patients.

\section{Sex Differences Directly Affect NLRP3 Inflammasome Activation}

Androgens are hormones that contribute to growth and reproduction in both men and women. Specifically, males tend to have higher androgen levels than females. Many studies have shown that androgen induces NLRP3 inflammasome activation in some disease conditions. For example, testosterone can directly activate NLRP3 inflammasome and mediate fibrosis in a CCl4-induced liver injury model (Ma et al., 2020). Excess testosterone production, which exceeds its normal range, can induce mitochondrial ROS to indirectly activate NLRP3 inflammasome (Alves et al., 2020). On the other hand, estrogen plays a more complicated role in regulating NLRP3 inflammasome activation. Specifically, estrogen induces NLRP3 inflammasome activation in tumor microenvironment (Wei et al., 2015; Liu 
et al., 2019; Wei et al., 2019), while inhibits this pathway under normal conditions. For instance, estrogen exhibits protective effects on brain by inhibiting NLRP3 inflammasome (Thakkar et al., 2016; Xu et al., 2016), and also demonstrates inhibitory effects at both mRNA and protein levels (Cheng et al., 2019). Besides, there are many factors that affect NLRP3 inflammasome activation (Zhang et al., 2020b), including ubiquitination and phosphorylation. The phosphokinases, such as JNK1, PKD and PKA, phosphorylate NLRP3 and promote its activation; while phosphatase, such as PP2A and PTPN22, mediate the dephosphorylation and suppression of NLRP3 (Song and Li, 2018). Estrogen downregulates receptor activator of nuclear factor-kappa B ligand (RANKL) to prevent RANKL-induced JNK activation (Srivastava et al., 2001), and its receptor directly binds to protein kinase D3 (PRKD3) gene promoter to decrease PKD3 expression (Borges et al., 2015), thus inhibiting phosphokinases-induced NLRP3 phosphorylation and activation. In addition, estrogen promotes the activity of PP2A (Ueda et al., 2013), thus leading to the dephosphorylation and impaired activation of NLRP3 inflammasome. Apart from estrogen, progesterone also inhibits NLRP3 inflammasome activation (Espinosa-Garcia et al., 2020). Notably, the levels of estrogen and progesterone decreased significantly after pausimenia (Harman et al., 2001; Galea et al., 2020), which in turn attenuated their protective effects. Furthermore, it has been reported that postmenopausal female patients with COVID-19 tend to share similar symptoms with male COVID-19 patients (Meng et al., 2020; Xie et al., 2020).

\section{Sex Differences Influence the Renin-Angiotensin-Aldosterone System to Activate NLRP3 Inflammasome}

In addition to the above-mentioned direct effects, sex hormones also affect the activation of NLRP3 inflammasome via the RAAS, especially during SARS-CoV-2 infection. ACE2 can act as a receptor to mediate the invasion and replication of SARS-CoV-2 virus. In addition, ACE2 also plays a central role in maintaining the homeostasis of RAAS. This system begins with angiotensin, which is converted to angiotensin I by renin, angiotensin I is then converted to angiotensin II by ACE, and finally angiotensin II is converted to angiotensin (1-7) by ACE2. Angiotensin II acts on AT1 and AT2 receptors, while angiotensin (1-7) acts on Mas receptor to neutralize angiotensin II/AT1R signal. The diminished level of ACE2 can break RAAS balance in angiotensin II/AT1R axis, while the increased level of ACE2 restores RAAS balance in angiotensin (1-7)/MASR axis to exert protective function (Patel et al., 2016).

In animal studies, ACE2 and AT2R have been shown to protect mice against ARDS caused by acid inhalation or sepsis, while other components of RAAS, such as ACE, angiotensin II and AT1R, can aggravate disease progression and lead to pulmonary edema and lung damage (Imai et al., 2005). The activation of ACE2/angiotensin (1-7)/MASR axis results in NLRP3 inflammasome inhibition, thus protecting from lipopolysaccharide-induced lung injury (Chen et al., 2019; Huang H. et al., 2020; Xu et al., 2020b; Zhao and Zhao, 2020). Furthermore, downregulation of ACE2 can lead to an imbalance of the RAAS and release of inflammatory cytokines (Mahmudpour et al., 2020).

During SARS virus infection, the level of ACE2 decreased significantly, while the level of ACE was not affected (Kuba et al., 2005), which made RAAS turn to angiotensin II/AT1R axis and elevated angiotensin II level can activate NLRP3 inflammasome (Zhang et al., 2016; Zhao et al., 2018; Wang et al., 2019), thus leading to development of ARDS. As similar to SARS-CoV, SARS-CoV-2 can also downregulate ACE2, and this downregulation is associated with disease severity (Kuba et al., 2005; Ahn et al., 2019; Zhang et al., 2020a). During SARS-CoV-2 infection, ACE2 interacts with SARS-CoV-2 spike protein to activate NLRP3 inflammasome, which in turn causes moderate inflammation (Ratajczak et al., 2020). A decrease in ACE2 inhibits the production of angiotensin (1-7), thereby exhibiting an inhibitory effect on NLRP3 inflammasome activation (Wen et al., 2016; Zhang et al., 2016; You et al., 2019).

Sex hormones regulate ACE2 expression to affect NLRP3 inflammasome activation. Both estrogen and androgen upregulate ACE2 expression (Bukowska et al., 2017; La Vignera et al., 2020). Male kidney ACE2 possesses higher activity than female (Liu et al., 2010), suggesting androgen possesses greater impact on ACE2 expression. However, RAS activation and angiotensin II upregulation are paralleled with renal injury in male, but not in female (Sullivan, 2008), suggesting that estrogen has a protective role in angiotensin II-induced kidney damage. Estrogen affects ACE-angiotensin II-AT1 axis by inhibiting ACE to reduce angiotensin II production (Bachmann et al., 1991; Brosnihan et al., 1999; Reckelhoff et al., 2000; Sullivan, 2008). In addition, estrogen decreases the expression of AT1R (Kisley et al., 1999; Wu et al., 2003) and increases the expression of ACE2, AT2R, MAS, angiotensin (1-7) and MASR (Cheng et al., 2015; Erfinanda et al., 2021). Hence, targeting SARS-CoV-2 infection-induced downregulation of ACE2 and angiotensin (1-7) as well as upregulation of angiotensin II can be an effective strategy to inhibit NLRP3 inflammasome activation.

\section{Sex Differences Regulate the Complement Cascade-induced NLRP3 Inflammasome Activation}

During SARS-CoV-2 infection, some secondary factors also affect NLRP3 inflammasome activation. Specifically, NLRP3 inflammasome is required for the complement cascademediated induction of caspase- 1 and IL- $1 \beta$ in intracerebral hemorrhage (Yao et al., 2017). The assembly of NLRP3 inflammasome requires the intracellular activation of $\mathrm{C} 5$ and stimulation of its receptor C5aR1 (Arbore et al., 2016), while $\mathrm{C} 5 \mathrm{aR} 2$ promotes the expression of protein kinase $\mathrm{R}$ and contributes to NLRP3 inflammasome activation (Yu et al., 2019). Complement membrane attack complexes (MAC) assembles NLRP3 inflammasome and trigger IL-1 activation in IFN- $\gamma$-primed human endothelium (Xie et al., 2019). All these findings suggest that the complement cascade is involved in the activation of NLRP3 inflammasome. 
NLRP3 inflammasome is activated in a complement-cascade dependent manner following MERS-CoV infection (Jiang et al., 2019). The increased levels of complement cascade components were found in COVID-19 patients, such as C5b-9, C4d and MASP2 (Magro et al., 2020), indicating that NLRP3 inflammasome may be activated in COVID-19 patients in a complement cascade-dependent manner.

Bacterial infection significantly upregulated the mRNA levels of complement cascade genes (C3-1, C3-3, Factor B and Factor $\mathrm{H}$ ) in trout liver, while $\mathrm{E} 2$ treatment inhibited the upregulation of these genes (Wenger et al., 2011), suggesting that E2 could inhibit the activation of complement cascade. Moreover, there are significant sex differences in the abundance and function of complement cascade in healthy individuals. For example, lower levels of C3 and properdin were found in female subjects, which are responsible for alternative complement cascade activation (Gaya da Costa et al., 2018). In addition, the pro-inflammatory effect of C5b-9 is inhibited during disease in female subjects (Kotimaa et al., 2016), suggesting the involvement of sex differences in complement cascade activation. Given that the complement cascade is a source of sexual dimorphism in vulnerability to diverse illnesses (Kamitaki et al., 2020), it is speculated this system may play a key role in regulating sex differences among COVID-19 patients.

\section{Sex Differences Regulate ATP-P2X7R-Mediated NLRP3 Inflammasome Activation}

During infection, ATP is released from the damaged cells to the extracellular environment. This extracellular ATP subsequently activates $\mathrm{P} 2 \mathrm{X} 7$ receptor and generates an ionic flux that activates NLRP3 inflammasome. This effect is generally mild and difficult to observe. However, ionic flux is a common mechanism shared by other PAMPs and DAMPs, which have no structural similarity (Gong et al., 2018). This property made ATPP2X7R signaling pathway synergistically interact with other NLRP3 inflammasome stimulus to initiate inflammatory responses. For example, gout arthritis is characterized by hyperuricemia-derived monosodium urate (MSU) deposition followed by NLRP3 inflammasome activation (Martinon et al., 2006), while MSU alone does not trigger gout flare. Further research showed that MSU synergizes with ATP to promote NLRP3 inflammasome activation and gout flare. Therefore, it is speculated that the function of P2X7R may determine whether hyperuricemia patients develop gout arthritis (Tao et al., 2013; Tao et al., 2017). In addition, there are differences in P2X7R functions among different individuals; thus, only hyperuricemia patients who have strong P2X7R function are prone to develop into gout. Based on this observation, COVID-19 patients who have strong P2X7R function may experience NLRP3 inflammasome overactivation and subsequently a "cytokine storm", which represents a major mechanism underlying the disease exacerbation in some but not all male patients. Therefore, P2X7R can serve as a potential target for COVID19 treatment.
During SARS-CoV-2 infection, testosterone level may be elevated because ACE-2 is expressed by Leydig cell and can be hijacked by the virus (Nashiry et al., 2021), which in turn leads to inflammatory pyroptosis in Leydig cells and increased extracellular ATP levels (Zhang et al., 2017). These extracellular ATPs activate P2X receptors expressed in Leydig cells (Antonio et al., 2009) and promote testosterone secretion (Foresta et al., 1996). Since testosterone exerts a promoting role in NLRP3 inflammasome activation, the elevated level of testosterone will further trigger NLRP3 inflammasome activation or even overactivation. In female subjects, estrogen impairs the function of P2X7R (Gorodeski, 2004), and inhibits the synergistic interaction between P2X7R and other NLRP3 inflammasome activating signals, thereby preventing NLRP3 inflammasome from overactivation. This may be an important reason why female COVID-19 patients are not likely to develop into severe disease.

In summary, sex hormones both directly and indirectly affect the activation of NLRP3 inflammasome through different mechanisms (Table 1). Besides, other mechanisms may also be involved. For example, heat shock protein 27 (HSP27) is a member of the small heat shock protein family that exerts extracellular anti-inflammatory effects (Batulan et al., 2016). A previous study has shown that HSP27 can alleviate SARS-CoV-2-induced cytokine storm by inhibiting NLRP3 inflammasome and other pathways (O'Brien and Sandhu, 2020). Since the extracellular release of HSP27 is largely dependent on estrogen levels (Rayner et al., 2008; Rayner et al., 2009), we speculate that estrogen can inhibit the activation of NLRP3 inflammasome by promoting HSP27 secretion.

\section{DISCUSSION}

Since NLRP3 inflammasome activation is regulated by many factors, the above-mentioned mechanisms may not be able to explain all of the details. However, it is sufficient to say that the sex-related overactivation of NLRP3 inflammasome increases overall mortality in male COVID-19 patients, and NLRP3 inflammasome is activated during coronavirus infection. Compared with other coronavirus infections, the mortality of COVID-19 caused by SARS-CoV-2 is relatively lower, indicating a moderate activation of NLRP3 inflammasome in COVID-19 patients. Under this condition, sex influence is amplified that leads to different mortality between male and female COVID-19 patients, while SARS and MERS patients are not influenced by sex differences.

Since NLRP3 inflammasome is well controlled in most patients with COVID-19, cytokine storm is not common. In light with this, molecular factors that affect the overactivation of NLRP3 inflammasome are vital for predicting the outcome of COVID-19 patients. For example, in addition to biological sex, obesity-associated NLRP3 inflammasome activating factors also increase the risk of cytokine storms (LópezReyes et al., 2020). Moreover, older adults possess higher levels of NLRP3 inflammasome (Stout-Delgado et al., 2016; 
TABLE 1 | Regulatory effects of sex hormones on NLRP3 inflammasome activation.

\begin{tabular}{|c|c|c|c|c|}
\hline & Influence way & Effect target & Activate/Inhibit & References \\
\hline \multirow[t]{8}{*}{ Estrogen } & ATP-P2X7R-NLRP3 & P2X7R & Inhibit & Gorodeski (2004) \\
\hline & ACE-angiotensin II-AT1-NLRP3 & ACE & Inhibit & Brosnihan et al. (1999), Kisley et al. (1999), Wu et al. (2003) \\
\hline & & AT1 & & \\
\hline & ACE2-angiotensin (1-7)-NLRP3 & ACE2 & Inhibit & Bukowska et al. (2017) \\
\hline & RANKL-JNK- NLRP3 phosphorylation & RANKL & Inhibit & Srivastava et al. (2001) \\
\hline & PKD3-NLRP3 phosphorylation & PKD3 & Inhibit & Borges et al. (2015) \\
\hline & PP2A-NLRP3 dephosphorylation & PP2A & Inhibit & Ueda et al. (2013) \\
\hline & HSP27-NLRP3 & HSP27 & Inhibit & Rayner et al. (2008), Rayner et al. (2009) \\
\hline Progesterone & HMGB1-NLRP3 & HMGB1 & Inhibit & Espinosa-Garcia et al. (2020) \\
\hline \multirow[t]{4}{*}{ Testosterone } & ROS-NLRP3 & ROS & Activate & Chignalia et al. (2012) \\
\hline & ATP-P2X7R-testosterone-NLRP3 & Testosterone & Activate & Foresta et al. (1996), Antonio et al. (2009), Zhang et al. (2017) \\
\hline & NLRP3 & NLRP3 & Activate & Ma et al. (2020) \\
\hline & mROS-NLRP3 & mROS & Activate & Alves et al. (2020) \\
\hline Androgen & ACE2-angiotensin (1-7)-NLRP3 & ACE2 & Inhibit & La Vignera et al. (2020) \\
\hline
\end{tabular}

Lara et al., 2020) and are prone to develop cytokine storms, thus elderly patient with COVID-19 have worse prognosis. Hence, for male, elderly, obese and high-risk COVID-19 patients with NLRP3 inflammasome overactivation, drugs that target NLRP3 inflammasome may be used to prevent disease progression from mild to severe. Growing evidence has shown that colchicine inhibits NLRP3 inflammasome activation (Demidowich et al., 2016) and effectively reduces the overall mortality of COVID-19 patients (Reyes et al., 2020).

In addition, the use of hydroxychloroquine in COVID-19 treatment is still controversial, as it failed to prevent SARSCoV-2 infection (Boulware et al., 2020). However, it do inhibit the activation of NLRP3 inflammasome (Tang et al., 2018). Considering the pivotal function of NLRP3 inflammasome in COVID-19, hydroxychloroquine could possibly inhibit NLRP3 inflammasome-induced cytokine storms and cause less severe symptoms (Lucchesi et al., 2020). Apart from colchicine and hydroxychloroquine, there are also other new or classic drugs that inhibit NLRP3 inflammasome and potentially effective to prevent the cytokine storms in COVID-19 patients (Zahid et al., 2019), including Tranilast and Oridonin. Furthermore, drugs like Anakinra that target the downstream effectors of NLRP3 inflammasome (such as IL-1 $\beta$, IL-18 and their receptors) may also exert protective effects against SARS-CoV-2 infection (Jamilloux et al., 2020; Pontali et al., 2020; Soy et al., 2020).

Other than COVID-19, there are also NLRP3 inflammasomeassociated diseases in which sex elements play a central role. For example, gout arthritis is characterized by MSUstimulated NLRP3 inflammasome activation. This disease can also be influenced by biological sex, which is more common in men than in women. Sex elements affect gout flare by regulating NLRP3 inflammasome activation and ATPP2X7R-NLRP3 pathway (Tao et al., 2013; Tao et al., 2017). Estrogen also inhibits the function of P2X7R (Gorodeski, 2004) to control gout flare, indicating the role of sex differences in this disease. However, inhibition of NLRP3 inflammasome in females is not always protective; in some cases, females are even more likely to develop infectious diseases. For example, Zika virus can activate NLRP3 inflammasome (Wang et al., 2018), and young women are more likely to be infected (Lozier et al., 2018). Given that the suppression of NLRP3 inflammasome by estrogen impairs the immune response against the virus and increases the risk of infection, this somehow explains that the majority of asymptomatic COVID-19 patients are females. Therefore, to avoid the damage caused by excessive inflammation, accurate detection of NLRP3 inflammasome is required to assess whether it exerts protective or detrimental role in SARSCoV-2 infection. At the same time, appropriate interventions should be developed for the treatment of this disease.

\section{AUTHOR CONTRIBUTIONS}

JT and $\mathrm{HZ}$ contributed to the conception and design of manuscript. HZ and YT drafted the manuscript. JT revised the manuscript. All authors reviewed and approved the final manuscript.

\section{FUNDING}

This work was supported by grants from National Natural Science Foundation of China (81771774), and the Anhui Key Research and Development Foundation (201904a07020103).

\section{ACKNOWLEDGMENTS}

The authors would like to express their gratitude to EditSprings (https://www.editsprings.com/) for the expert linguistic services provided, and thank Ayesha Zahid for her language editing of the manuscript. 


\section{REFERENCES}

Ahn, M., Anderson, D. E., Zhang, Q., Tan, C. W., Lim, B. L., Luko, K., et al. (2019). Dampened NLRP3-Mediated Inflammation in Bats and Implications for a Special Viral Reservoir Host. Nat. Microbiol. 4 (5), 789-799. doi:10.1038/ s41564-019-0371-3

Alves, J. V., da Costa, R. M., Pereira, C. A., Fedoce, A. G., Silva, C. A. A., Carneiro, F. S., et al. (2020). Supraphysiological Levels of Testosterone Induce Vascular Dysfunction via Activation of the NLRP3 Inflammasome. Front. Immunol. 11, 1647. doi:10.3389/fimmu.2020.01647

Antonio, L. S., Costa, R. R., Gomes, M. D., and Varanda, W. A. (2009). Mouse Leydig Cells Express Multiple P2X Receptor Subunits. Purinergic Signal. 5 (3), 277-287. doi:10.1007/s11302-008-9128-9

Arbore, G., West, E. E., Spolski, R., Robertson, A. A. B., Klos, A., Rheinheimer, C., et al. (2016). T Helper 1 Immunity Requires Complement-Driven NLRP3 Inflammasome Activity in CD4+ T Cells. Science 352 (6292), aad1210. doi:10. 1126/science.aad 1210

Bachmann, J., Feldmer, M., Ganten, U., Stock, G., and Ganten, D. (1991). Sexual Dimorphism of Blood Pressure: Possible Role of the Renin-Angiotensin System. J. Steroid Biochem. Mol. Biol. 40 (4-6), 511-515. doi:10.1016/0960-0760(91) 90270-f

Batulan, Z., Pulakazhi Venu, V. K., Li, Y., Koumbadinga, G., Alvarez-Olmedo, D. G., Shi, C., et al. (2016). Extracellular Release and Signaling by Heat Shock Protein 27: Role in Modifying Vascular Inflammation. Front. Immunol. 7, 285. doi:10.3389/fimmu.2016.00285

Borges, S., Perez, E. A., Thompson, E. A., Radisky, D. C., Geiger, X. J., and Storz, P. (2015). Effective Targeting of Estrogen Receptor-Negative Breast Cancers with the Protein Kinase D Inhibitor CRT0066101. Mol. Cancer Ther. 14 (6), 1306-1316. doi:10.1158/1535-7163.Mct-14-0945

Boulware, D. R., Pullen, M. F., Bangdiwala, A. S., Pastick, K. A., Lofgren, S. M., Okafor, E. C., et al. (2020). A Randomized Trial of Hydroxychloroquine as Postexposure Prophylaxis for Covid-19. N. Engl. J. Med. 383 (6), 517-525. doi:10.1056/NEJMoa2016638

Brosnihan, K. B., Senanayake, P. S., Li, P., and Ferrario, C. M. (1999). Bi-directional Actions of Estrogen on the Renin-Angiotensin System. Braz. J. Med. Biol. Res. 32 (4), 373-381. doi:10.1590/s0100-879x1999000400001

Bukowska, A., Spiller, L., Wolke, C., Lendeckel, U., Weinert, S., Hoffmann, J., et al. (2017). Protective Regulation of the ACE2/ACE Gene Expression by Estrogen in Human Atrial Tissue from Elderly Men. Exp. Biol. Med. (Maywood) 242 (14), 1412-1423. doi:10.1177/1535370217718808

Caricchio, R., Gallucci, M., Dass, C., Zhang, X., Gallucci, S., Fleece, D., et al. (2021). Preliminary Predictive Criteria for COVID-19 Cytokine Storm. Ann. Rheum. Dis. 80 (1), 88-95. doi:10.1136/annrheumdis-2020-218323

Chang, Y.-S., Ko, B.-H., Ju, J.-C., Chang, H.-H., Huang, S.-H., and Lin, C.-W. (2020). SARS Unique Domain (SUD) of Severe Acute Respiratory Syndrome Coronavirus Induces NLRP3 Inflammasome-dependent CXCL10-Mediated Pulmonary Inflammation. Ijms 21 (9), 3179. doi:10.3390/ijms21093179

Chen, I.-Y., Moriyama, M., Chang, M.-F., and Ichinohe, T. (2019). Severe Acute Respiratory Syndrome Coronavirus Viroporin 3a Activates the NLRP3 Inflammasome. Front. Microbiol. 10, 50. doi:10.3389/fmicb.2019.00050

Chen, N., Zhou, M., Dong, X., Qu, J., Gong, F., Han, Y., et al. (2020). Epidemiological and Clinical Characteristics of 99 Cases of 2019 Novel Coronavirus Pneumonia in Wuhan, China: a Descriptive Study. The Lancet 395 (10223), 507-513. doi:10.1016/s0140-6736(20)30211-7

Cheng, C., Wu, H., Wang, M., Wang, L., Zou, H., Li, S., et al. (2019). Estrogen Ameliorates Allergic Airway Inflammation by Regulating Activation of NLRP3 in Mice. Biosci. Rep. 39 (1). doi:10.1042/bsr20181117

Cheng, Y., Li, Q., Zhang, Y., Wen, Q., and Zhao, J. (2015). Effects of Female Sex Hormones on Expression of the Ang-(1-7)/Mas-R/nNOS Pathways in Rat Brain. Can. J. Physiol. Pharmacol. 93 (11), 993-998. doi:10.1139/cjpp-20150087

Chignalia, A. Z., Schuldt, E. Z., Camargo, L. L., Montezano, A. C., Callera, G. E., Laurindo, F. R., et al. (2012). Testosterone Induces Vascular Smooth Muscle Cell Migration by NADPH Oxidase and c-Src-Dependent Pathways. Hypertension 59 (6), 1263-1271. doi:10.1161/hypertensionaha. 111.180620 de Rivero Vaccari, J. C., Dietrich, W. D., Keane, R. W., and de Rivero Vaccari, J. P. (2020). The Inflammasome in Times of COVID-19. Front. Immunol. 11, 583373. doi:10.3389/fimmu.2020.583373

Demidowich, A. P., Davis, A. I., Dedhia, N., and Yanovski, J. A. (2016). Colchicine to Decrease NLRP3-Activated Inflammation and Improve Obesity-Related Metabolic Dysregulation. Med. Hypotheses 92, 67-73. doi:10.1016/j.mehy. 2016.04.039

Dimopoulos, G., de Mast, Q., Markou, N., Theodorakopoulou, M., Komnos, A. Mouktaroudi, M., et al. (2020). Favorable Anakinra Responses in Severe Covid19 Patients with Secondary Hemophagocytic Lymphohistiocytosis. Cell Host \& Microbe 28 (1), 117-123. doi:10.1016/j.chom.2020.05.007

Erfinanda, L., Ravindran, K., Kohse, F., Gallo, K., Preissner, R., Walther, T., et al. (2021). Oestrogen-mediated Upregulation of the Mas Receptor Contributes to Sex Differences in Acute Lung Injury and Lung Vascular Barrier Regulation. Eur. Respir. J. 57 (1), 2000921. doi:10.1183/13993003. 00921-2020

Espinosa-Garcia, C., Atif, F., Yousuf, S., Sayeed, I., Neigh, G. N., and Stein, D. G. (2020). Progesterone Attenuates Stress-Induced NLRP3 Inflammasome Activation and Enhances Autophagy Following Ischemic Brain Injury. Ijms 21 (11), 3740. doi:10.3390/ijms21113740

Foresta, C., Rossato, M., Nogara, A., Gottardello, F., Bordon, P., and Di Virgilio, F. (1996). Role of P2-Purinergic Receptors in Rat Leydig Cell Steroidogenesis. Biochem. J. 320 (Pt 2Pt 2), 499-504. doi:10.1042/bj3200499

Freeman, T. L., and Swartz, T. H. (2020). Targeting the NLRP3 Inflammasome in Severe COVID-19. Front. Immunol. 11, 1518. doi:10.3389/fimmu.2020. 01518

Galea, L. A. M., Choleris, E., Albert, A. Y. K., McCarthy, M. M., and Sohrabji, F. (2020). The Promises and Pitfalls of Sex Difference Research. Front. Neuroendocrinology 56, 100817. doi:10.1016/j.yfrne.2019.100817

Ganter, M. T., Roux, J., Miyazawa, B., Howard, M., Frank, J. A., Su, G., et al. (2008). Interleukin-1 $\beta$ Causes Acute Lung Injury via $\alpha v \beta 5$ and $\alpha v \beta 6$ Integrindependent Mechanisms. Circ. Res. 102 (7), 804-812. doi:10.1161/circresaha. 107.161067

Gaya da Costa, M., Poppelaars, F., van Kooten, C., Mollnes, T. E., Tedesco, F., Würzner, R., et al. (2018). Age and Sex-Associated Changes of Complement Activity and Complement Levels in a Healthy Caucasian Population. Front. Immunol. 9, 2664. doi:10.3389/fimmu.2018.02664

Gebhard, C., Regitz-Zagrosek, V., Neuhauser, H. K., Morgan, R., and Klein, S. L. (2020). Impact of Sex and Gender on COVID-19 Outcomes in Europe. Biol. Sex. Differ. 11 (1), 29. doi:10.1186/s13293-020-00304-9

Gong, T., Yang, Y., Jin, T., Jiang, W., and Zhou, R. (2018). Orchestration of NLRP3 Inflammasome Activation by Ion Fluxes. Trends Immunol. 39 (5), 393-406. doi:10.1016/j.it.2018.01.009

Gorodeski, G. I. (2004). Estrogen Attenuates P2X7-R-Mediated Apoptosis of Uterine Cervical Cells by Blocking Calcium Influx. Nucleosides, Nucleotides and Nucleic Acids 23 (8-9), 1287-1293. doi:10.1081/NCN-200027549

Harman, S. M., Metter, E. J., Tobin, J. D., Pearson, J., and Blackman, M. R. (2001). Longitudinal Effects of Aging on Serum Total and Free Testosterone Levels in Healthy Men. J. Clin. Endocrinol. Metab. 86 (2), 724-731. doi:10.1210/jcem.86. 2.7219

Huang, C., Wang, Y., Li, X., Ren, L., Zhao, J., Hu, Y., et al. (2020a). Clinical Features of Patients Infected with 2019 Novel Coronavirus in Wuhan, China. The Lancet 395 (10223), 497-506. doi:10.1016/s0140-6736(20)30183-5

Huang, H., Wang, J., Liu, Z., and Gao, F. (2020b). The Angiotensin-Converting Enzyme 2/angiotensin (1-7)/mas axis Protects against Pyroptosis in LPSInduced Lung Injury by Inhibiting NLRP3 Activation. Arch. Biochem. Biophys. 693, 108562. doi:10.1016/j.abb.2020.108562

Imai, Y., Kuba, K., Rao, S., Huan, Y., Guo, F., Guan, B., et al. (2005). Angiotensinconverting Enzyme 2 Protects from Severe Acute Lung Failure. Nature 436 (7047), 112-116. doi:10.1038/nature03712

Jamilloux, Y., Henry, T., Belot, A., Viel, S., Fauter, M., El Jammal, T., et al. (2020). Should We Stimulate or Suppress Immune Responses in COVID-19? Cytokine and Anti-cytokine Interventions. Autoimmun. Rev. 19 (7), 102567. doi:10.1016/ j.autrev.2020.102567

Jiang, Y., Li, J., Teng, Y., Sun, H., Tian, G., He, L., et al. (2019). Complement Receptor C5aR1 Inhibition Reduces Pyroptosis in hDPP4-Transgenic Mice Infected with MERS-CoV. Viruses 11 (1), 39. doi:10.3390/v11010039 
Kamitaki, N., Sekar, A., Sekar, A., Handsaker, R. E., de Rivera, H., Tooley, K., et al. (2020). Complement Genes Contribute Sex-Biased Vulnerability in Diverse Disorders. Nature 582 (7813), 577-581. doi:10.1038/s41586-020-2277-x

Kim, D., Lee, J.-Y., Yang, J.-S., Kim, J. W., Kim, V. N., and Chang, H. (2020). The Architecture of SARS-CoV-2 Transcriptome. Cell 181 (4), 914-921. doi:10. 1016/j.cell.2020.04.011

Kisley, L. R., Sakai, R. R., and Fluharty, S. J. (1999). Estrogen Decreases Hypothalamic Angiotensin II AT1 Receptor Binding and mRNA in the Female Rat. Brain Res. 844 (1-2), 34-42. doi:10.1016/s0006-8993(99)01815-6

Klein, S. L., Dhakal, S., Ursin, R. L., Deshpande, S., Sandberg, K., and MauvaisJarvis, F. (2020). Biological Sex Impacts COVID-19 Outcomes. Plos Pathog. 16 (6), e1008570. doi:10.1371/journal.ppat.1008570

Kolb, M., Margetts, P. J., Anthony, D. C., Pitossi, F., and Gauldie, J. (2001). Transient Expression of IL-1 $\beta$ Induces Acute Lung Injury and Chronic Repair Leading to Pulmonary Fibrosis. J. Clin. Invest. 107 (12), 1529-1536. doi:10. $1172 /$ jci12568

Kotimaa, J., Klar-Mohammad, N., Gueler, F., Schilders, G., Jansen, A., Rutjes, H., et al. (2016). Sex Matters: Systemic Complement Activity of Female C57BL/6J and BALB/cJ Mice Is Limited by Serum Terminal Pathway Components. Mol. Immunol. 76, 13-21. doi:10.1016/j.molimm.2016.06.004

Kuba, K., Imai, Y., Rao, S., Gao, H., Guo, F., Guan, B., et al. (2005). A Crucial Role of Angiotensin Converting Enzyme 2 (ACE2) in SARS Coronavirus-Induced Lung Injury. Nat. Med. 11 (8), 875-879. doi:10.1038/nm1267

La Vignera, S., Cannarella, R., Condorelli, R. A., Torre, F., Aversa, A., and Calogero, A. E. (2020). Sex-Specific SARS-CoV-2 Mortality: Among HormoneModulated ACE2 Expression, Risk of Venous Thromboembolism and Hypovitaminosis D. Ijms 21 (8), 2948. doi:10.3390/ijms21082948

Lara, P. C., Macías-Verde, D., and Burgos-Burgos, J. (2020). Age-induced NLRP3 Inflammasome Over-activation Increases Lethality of SARS-CoV-2 Pneumonia in Elderly Patients. Aging Dis. 11 (4), 756-762. doi:10.14336/ad.2020.0601

Li, Q., Guan, X., Wu, P., Wang, X., Zhou, L., Tong, Y., et al. (2020). Early Transmission Dynamics in Wuhan, China, of Novel Coronavirus-Infected Pneumonia. N. Engl. J. Med. 382 (13), 1199-1207. doi:10.1056/ NEJMoa2001316

Lin, L., Xu, L., Lv, W., Han, L., Xiang, Y., Fu, L., et al. (2019). An NLRP3 Inflammasome-Triggered Cytokine Storm Contributes to Streptococcal Toxic Shock-like Syndrome (STSLS). Plos Pathog. 15 (6), e1007795. doi:10.1371/journal.ppat.1007795

Liu, J., Ji, H., Zheng, W., Wu, X., Zhu, J. J., Arnold, A. P., et al. (2010). Sex Differences in Renal Angiotensin Converting Enzyme 2 (ACE2) Activity Are $17 \beta$-oestradiol-dependent and Sex Chromosome-independent. Biol. Sex Dif 1 (1), 6. doi:10.1186/2042-6410-1-6

Liu, S.-G., Wu, X.-X., Hua, T., Xin, X.-y., Feng, D.-L., Chi, S.-Q., et al. (2019). NLRP3 Inflammasome Activation by Estrogen Promotes the Progression of Human Endometrial Cancer. Ott Vol. 12, 6927-6936. doi:10.2147/ott.S218240

López-Reyes, A., Martinez-Armenta, C., Espinosa-Velázquez, R., VázquezCárdenas, P., Cruz-Ramos, M., Palacios-Gonzalez, B., et al. (2020). NLRP3 Inflammasome: The Stormy Link between Obesity and COVID-19. Front. Immunol. 11, 570251. doi:10.3389/fimmu.2020.570251

Lozier, M. J., Burke, R. M., Lopez, J., Acevedo, V., Amador, M., Read, J. S., et al. (2018). Differences in Prevalence of Symptomatic Zika Virus Infection, by Age and SexPuerto Rico, 2016. J. Infect. Dis. 217 (11), 1678-1689. doi:10.1093/infdis/jix630

Lucchesi, A., Silimbani, P., Musuraca, G., Cerchione, C., Martinelli, G., Di Carlo, P., et al. (2020). Clinical and Biological Data on the Use of Hydroxychloroquine against SARS-CoV-2 Could Support the Role of the NLRP3 Inflammasome in the Pathogenesis of Respiratory Disease. J. Med. Virol. 93, 124-126. doi:10. 1002/jmv. 26217

Ma, X., Zhou, Y., Qiao, B., Jiang, S., Shen, Q., Han, Y., et al. (2020). Androgen Aggravates Liver Fibrosis by Activation of NLRP3 Inflammasome in CCl4Induced Liver Injury Mouse Model. Am. J. Physiology-Endocrinology Metab. 318 (5), E817-e829. doi:10.1152/ajpendo.00427.2019

Magro, C., Mulvey, J. J., Berlin, D., Nuovo, G., Salvatore, S., Harp, J., et al. (2020). Complement Associated Microvascular Injury and Thrombosis in the Pathogenesis of Severe COVID-19 Infection: A Report of Five Cases. Translational Res. 220, 1-13. doi:10.1016/j.trsl.2020.04.007

Mahmudpour, M., Roozbeh, J., Keshavarz, M., Farrokhi, S., and Nabipour, I. (2020). COVID-19 Cytokine Storm: The Anger of Inflammation. Cytokine 133, 155151. doi:10.1016/j.cyto.2020.155151
Martinon, F., Pétrilli, V., Mayor, A., Tardivel, A., and Tschopp, J. (2006). Goutassociated Uric Acid Crystals Activate the NALP3 Inflammasome. Nature 440 (7081), 237-241. doi:10.1038/nature04516

Meng, Y., Wu, P., Lu, W., Liu, K., Ma, K., Huang, L., et al. (2020). Sex-specific Clinical Characteristics and Prognosis of Coronavirus Disease-19 Infection in Wuhan, China: A Retrospective Study of 168 Severe Patients. Plos Pathog. 16 (4), e1008520. doi:10.1371/journal.ppat.1008520

Nashiry, A., Sarmin Sumi, S., Islam, S., Quinn, J. M. W., and Moni, M. A. (2021). Bioinformatics and System Biology Approach to Identify the Influences of COVID-19 on Cardiovascular and Hypertensive Comorbidities. Brief Bioinform 22, 1387-1401. doi:10.1093/bib/bbaa426

Nieto-Torres, J. L., Verdiá-Báguena, C., Jimenez-Guardeño, J. M., Regla-Nava, J. A., Castaño-Rodriguez, C., Fernandez-Delgado, R., et al. (2015). Severe Acute Respiratory Syndrome Coronavirus E Protein Transports Calcium Ions and Activates the NLRP3 Inflammasome. Virology 485, 330-339. doi:10.1016/j. virol.2015.08.010

O'Brien, E. R., and Sandhu, J. K. (2020). Sex Differences in COVID-19 Mortality: Opportunity to Develop HSP27 (HSPB1) Immunotherapy to Treat HyperInflammation? Cell Stress Chaperones 25 (5), 725-729. doi:10.1007/s12192-02001146-5

Pan, P., Shen, M., Yu, Z., Ge, W., Chen, K., Tian, M., et al. (2020). SARS-CoV-2 N Promotes the NLRP3 Inflammasome Activation to Induce Hyperinflammation. doi:10.21203/rs.3.rs-101224/v1

Patel, V. B., Zhong, J.-C., Grant, M. B., and Oudit, G. Y. (2016). Role of the ACE2/ Angiotensin 1-7 Axis of the Renin-Angiotensin System in Heart Failure. Circ. Res. 118 (8), 1313-1326. doi:10.1161/circresaha.116.307708

Pontali, E., Volpi, S., Antonucci, G., Castellaneta, M., Buzzi, D., Tricerri, F., et al. (2020). Safety and Efficacy of Early High-Dose IV Anakinra in Severe COVID19 Lung Disease. J. Allergy Clin. Immunol. 146 (1), 213-215. doi:10.1016/j.jaci. 2020.05.002

Ratajczak, M. Z., Bujko, K., Ciechanowicz, A., Sielatycka, K., Cymer, M., Marlicz, W., et al. (2020). SARS-CoV-2 Entry Receptor ACE2 Is Expressed on Very Small CD45- Precursors of Hematopoietic and Endothelial Cells and in Response to Virus Spike Protein Activates the Nlrp3 Inflammasome. Stem Cel Rev Rep. 17, 266-277. doi:10.1007/s12015-020-10010-z

Ratajczak, M. Z., and Kucia, M. (2020). SARS-CoV-2 Infection and Overactivation of Nlrp3 Inflammasome as a Trigger of Cytokine "storm" and Risk Factor for Damage of Hematopoietic Stem Cells. Leukemia 34 (7), 1726-1729. doi:10. 1038/s41375-020-0887-9

Rayner, K., Chen, Y.-X., McNulty, M., Simard, T., Zhao, X., Wells, D. J., et al. (2008). Extracellular Release of the Atheroprotective Heat Shock Protein 27 Is Mediated by Estrogen and Competitively Inhibits acLDL Binding to Scavenger Receptor-A. Circ. Res. 103 (2), 133-141. doi:10.1161/circresaha. 108.172155

Rayner, K., Sun, J., Chen, Y.-X., McNulty, M., Simard, T., Zhao, X., et al. (2009). Heat Shock Protein 27 Protects against Atherogenesis via an Estrogendependent Mechanism. Atvb 29 (11), 1751-1756. doi:10.1161/atvbaha.109. 193656

Reckelhoff, J. F., Zhang, H., and Srivastava, K. (2000). Gender Differences in Development of Hypertension in Spontaneously Hypertensive Rats. Hypertension 35 (1 Pt 2), 480-483. doi:10.1161/01.hyp.35.1.480

Reyes, A. Z., Hu, K. A., Teperman, J., Wampler Muskardin, T. L., Tardif, J.-C., Shah, B., et al. (2020). Anti-inflammatory Therapy for COVID-19 Infection: the Case for Colchicine. Ann. Rheum. Dis. 80, 550-557. doi:10.1136/annrheumdis2020-219174

Rizk, J. G., Kalantar-Zadeh, K., Mehra, M. R., Lavie, C. J., Rizk, Y., and Forthal, D. N. (2020). Pharmaco-Immunomodulatory Therapy in COVID-19. Drugs 80 (13), 1267-1292. doi:10.1007/s40265-020-01367-z

Rodrigues, T. S., de Sá, K. S. G., Ishimoto, A. Y., Becerra, A., Oliveira, S., Almeida, L., et al. (2021). Inflammasomes Are Activated in Response to SARS-CoV-2 Infection and Are Associated with COVID-19 Severity in Patients. J. Exp. Med. 218 (3). doi:10.1084/jem.20201707

Schroder, K., and Tschopp, J. (2010). The Inflammasomes. Cell 140 (6), 821-832. doi:10.1016/j.cell.2010.01.040

Shi, C.-S., Nabar, N. R., Huang, N.-N., and Kehrl, J. H. (2019). SARS-coronavirus Open Reading Frame-8b Triggers Intracellular Stress Pathways and Activates NLRP3 Inflammasomes. Cell Death Discov. 5, 101. doi:10.1038/s41420-0190181-7 
Siu, K. L., Yuen, K. S., Castano-Rodriguez, C., Ye, Z. W., Yeung, M. L., Fung, S. Y., et al. (2019). Severe Acute Respiratory Syndrome Coronavirus ORF3a Protein Activates the NLRP3 Inflammasome by Promoting TRAF3dependent Ubiquitination of ASC. FASEB j. 33 (8), 8865-8877. doi:10. 1096/fj.201802418R

Song, N., and Li, T. (2018). Regulation of NLRP3 Inflammasome by Phosphorylation. Front. Immunol. 9, 2305. doi:10.3389/fimmu.2018.02305

Soy, M., Keser, G., Atagündüz, P., Tabak, F., Atagündüz, I., and Kayhan, S. (2020). Cytokine Storm in COVID-19: Pathogenesis and Overview of Antiinflammatory Agents Used in Treatment. Clin. Rheumatol. 39 (7), 2085-2094. doi:10.1007/s10067-020-05190-5

Srivastava, S., Toraldo, G., Weitzmann, M. N., Cenci, S., Ross, F. P., and Pacifici, R. (2001). Estrogen Decreases Osteoclast Formation by DownRegulating Receptor Activator of NF-Kb Ligand (RANKL)-induced JNK Activation. J. Biol. Chem. 276 (12), 8836-8840. doi:10.1074/jbc. M010764200

Stout-Delgado, H. W., Cho, S. J., Chu, S. G., Mitzel, D. N., Villalba, J., El-Chemaly, S., et al. (2016). Age-Dependent Susceptibility to Pulmonary Fibrosis Is Associated with NLRP3 Inflammasome Activation. Am. J. Respir. Cel Mol Biol. 55 (2), 252-263. doi:10.1165/rcmb.2015-0222OC

Sullivan, J. C. (2008). Sex and the Renin-Angiotensin System: Inequality between the Sexes in Response to RAS Stimulation and Inhibition. Am. J. PhysiologyRegulatory, Integr. Comp. Physiol. 294 (4), R1220-R1226. doi:10.1152/ajpregu. 00864.2007

Takahashi, T., Ellingson, M. K., Ellingson, M. K., Wong, P., Israelow, B., Lucas, C., et al. (2020). Sex Differences in Immune Responses that Underlie COVID-19 Disease Outcomes. Nature 588 (7837), 315-320. doi:10.1038/s41586-0202700-3

Tang, T.-T., Lv, L.-L., Pan, M.-M., Wen, Y., Wang, B., Li, Z.-L., et al. (2018). Hydroxychloroquine Attenuates Renal Ischemia/reperfusion Injury by Inhibiting Cathepsin Mediated NLRP3 Inflammasome Activation. Cell Death Dis 9 (3), 351. doi:10.1038/s41419-018-0378-3

Tao, J.-H., Cheng, M., Tang, J.-P., Dai, X.-J., Zhang, Y., Li, X.-P., et al. (2017). Single Nucleotide Polymorphisms Associated with P2X7R Function Regulate the Onset of Gouty Arthritis. PLoS One 12 (8), e0181685. doi:10.1371/journal. pone. 0181685

Tao, J.-H., Zhang, Y., and Li, X.-P. (2013). P2X7R: a Potential Key Regulator of Acute Gouty Arthritis. Semin. Arthritis Rheum. 43 (3), 376-380. doi:10.1016/j. semarthrit.2013.04.007

Tate, M. D., Ong, J. D. H., Dowling, J. K., McAuley, J. L., Robertson, A. B., Latz, E., et al. (2016). Reassessing the Role of the NLRP3 Inflammasome during Pathogenic Influenza A Virus Infection via Temporal Inhibition. Sci. Rep. 6, 27912. doi:10.1038/srep27912

Thakkar, R., Wang, R., Sareddy, G., Wang, J., Thiruvaiyaru, D., Vadlamudi, R., et al. (2016). NLRP3 Inflammasome Activation in the Brain after Global Cerebral Ischemia and Regulation by $17 \beta$-Estradiol. Oxidative Med. Cell Longevity 2016, 1-17. doi:10.1155/2016/8309031

Theobald, S. J., Simonis, A., Kreer, C., Zehner, M., Fischer, J., Albert, M.-C., et al. (2020). The SARS-CoV-2 Spike Protein Primes Inflammasome-Mediated Interleukin-1- Beta Secretion in COVID-19 Patient-Derived Macrophages. doi:10.21203/rs.3.rs-30407/v1

Ueda, K., Lu, Q., Baur, W., Aronovitz, M. J., and Karas, R. H. (2013). Rapid Estrogen Receptor Signaling Mediates Estrogen-Induced Inhibition of Vascular Smooth Muscle Cell Proliferation. Arterioscler Thromb. Vasc. Biol. 33 (8), 1837-1843. doi:10.1161/atvbaha.112.300752

van den Berg, D. F., and Te Velde, A. A. (2020). Severe COVID-19: NLRP3 Inflammasome Dysregulated. Front. Immunol. 11, 1580. doi:10.3389/fimmu. 2020.01580

Wang, J., Feng, Y., Huo, H., Zhang, X., Yue, J., Zhang, W., et al. (2019). NLRP3 Inflammasome Mediates Angiotensin II-Induced Islet $\beta$ Cell Apoptosis. Acta Biochim. Biophys. Sin (Shanghai) 51 (5), 501-508. doi:10.1093/abbs/ gmz032

Wang, W., Li, G., De Wu, W., Luo, Z., Pan, P., Tian, M., et al. (2018). Zika Virus Infection Induces Host Inflammatory Responses by Facilitating NLRP3 Inflammasome Assembly and Interleukin- $1 \beta$ Secretion. Nat. Commun. 9 (1), 106. doi:10.1038/s41467-017-02645-3

Wei, Q., Guo, P., Mu, K., Zhang, Y., Zhao, W., Huai, W., et al. (2015). Estrogen Suppresses Hepatocellular Carcinoma Cells through ER $\beta$-Mediated
Upregulation of the NLRP3 Inflammasome. Lab. Invest. 95 (7), 804-816. doi:10.1038/labinvest.2015.63

Wei, Q., Zhu, R., Zhu, J., Zhao, R., and Li, M. (2019). E2-Induced Activation of the NLRP3 Inflammasome Triggers Pyroptosis and Inhibits Autophagy in HCC Cells. Oncol. Res. 27 (7), 827-834. doi:10.3727/ 096504018x15462920753012

Wen, Y., Liu, Y., Tang, T., Lv, L., Liu, H., Ma, K., et al. (2016). NLRP3 Inflammasome Activation Is Involved in Ang II-Induced Kidney Damage via Mitochondrial Dysfunction. Oncotarget 7 (34), 54290-54302. doi:10. 18632/oncotarget.11091

Wenger, M., Sattler, U., Goldschmidt-Clermont, E., and Segner, H. (2011). 17Betaestradiol Affects the Response of Complement Components and Survival of Rainbow trout (Oncorhynchus mykiss) Challenged by Bacterial Infection. Fish Shellfish Immunol. 31 (1), 90-97. doi:10.1016/j.fsi.2011.04.007

Wu, X., Cakmak, S., Wortmann, M., Hakimi, M., Zhang, J., Böckler, D., et al. (2016). Sex- and Disease-specific Inflammasome Signatures in Circulating Blood Leukocytes of Patients with Abdominal Aortic Aneurysm. Mol. Med. 22, 508-518. doi:10.2119/molmed.2016.00035

Wu, Z., Maric, C., Roesch, D. M., Zheng, W., Verbalis, J. G., and Sandberg, K. (2003). Estrogen Regulates Adrenal Angiotensin AT1Receptors by Modulating AT1Receptor Translation. Endocrinology 144 (7), 3251-3261. doi:10.1210/en. 2003-0015

Xie, C. B., Qin, L., Li, G., Fang, C., Kirkiles-Smith, N. C., Tellides, G., et al. (2019). Complement Membrane Attack Complexes Assemble NLRP3 Inflammasomes Triggering IL-1 Activation of IFN- $\gamma$-Primed Human Endothelium. Circ. Res. 124 (12), 1747-1759. doi:10.1161/circresaha.119.314845

Xie, J., Tong, Z., Guan, X., Du, B., and Qiu, H. (2020). Clinical Characteristics of Patients Who Died of Coronavirus Disease 2019 in China. JAMA Netw. Open 3 (4), e205619, doi:10.1001/jamanetworkopen.2020.5619

Xu, H., Chitre, S. A., Akinyemi, I. A., Loeb, J. C., Lednicky, J. A., McIntosh, M. T., et al. (2020a). SARS-CoV-2 Viroporin Triggers the NLRP3 Inflammatory Pathway. doi:10.1101/2020.10.27.357731

Xu, H., Zhong, L., Deng, J., Peng, J., Dan, H., Zeng, X., et al. (2020b). High Expression of ACE2 Receptor of 2019-nCoV on the Epithelial Cells of Oral Mucosa. Int. J. Oral Sci. 12 (1), 8. doi:10.1038/s41368-020-0074-x

Xu, X., Han, M., Li, T., Sun, W., Wang, D., Fu, B., et al. (2020c). Effective Treatment of Severe COVID-19 Patients with Tocilizumab. Proc. Natl. Acad. Sci. USA 117 (20), 10970-10975. doi:10.1073/pnas.2005615117

$\mathrm{Xu}$, Y., Sheng, H., Bao, Q., Wang, Y., Lu, J., and Ni, X. (2016). NLRP3 Inflammasome Activation Mediates Estrogen Deficiency-Induced Depression- and Anxiety-like Behavior and Hippocampal Inflammation in Mice. Brain Behav. Immun. 56, 175-186. doi:10.1016/j.bbi.2016.02.022

Yang, Y., Wang, H., Kouadir, M., Song, H., and Shi, F. (2019). Recent Advances in the Mechanisms of NLRP3 Inflammasome Activation and its Inhibitors. Cel Death Dis 10 (2), 128. doi:10.1038/s41419-019-1413-8

Yao, S.-T., Cao, F., Chen, J.-L., Chen, W., Fan, R.-M., Li, G., et al. (2017). NLRP3 Is Required for Complement-Mediated Caspase-1 and IL-1beta Activation in ICH. J. Mol. Neurosci. 61 (3), 385-395. doi:10.1007/s12031-016-0874-9

You, Y., Huang, Y., Wang, D., Li, Y., Wang, G., Jin, S., et al. (2019). Angiotensin (17) Inhibits Arecoline-Induced Migration and Collagen Synthesis in Human Oral Myofibroblasts via Inhibiting NLRP3 Inflammas Ome Activation. J. Cel Physiol 234 (4), 4668-4680. doi:10.1002/jcp.27267

Yu, S., Wang, D., Huang, L., Zhang, Y., Luo, R., Adah, D., et al. (2019). The Complement Receptor C5aR2 Promotes Protein Kinase R Expression and Contributes to NLRP3 Inflammasome Activation and HMGB1 Release from Macrophages. J. Biol. Chem. 294 (21), 8384-8394. doi:10.1074/jbc.RA118. 006508

Zahid, A., Li, B., Kombe, A. J. K., Jin, T., and Tao, J. (2019). Pharmacological Inhibitors of the NLRP3 Inflammasome. Front. Immunol. 10, 2538. doi:10. 3389/fimmu.2019.02538

Zhang, C., He, H., Wang, L., Zhang, N., Huang, H., Xiong, Q., et al. (2017). VirusTriggered ATP Release Limits Viral Replication through Facilitating IFN- $\beta$ Production in a P2X7-dependent Manner. J.I. 199 (4), 1372-1381. doi:10.4049/ jimmunol.1700187

Zhang, H., Penninger, J. M., Li, Y., Zhong, N., and Slutsky, A. S. (2020a). Angiotensin-converting Enzyme 2 (ACE2) as a SARS-CoV-2 Receptor: Molecular Mechanisms and Potential Therapeutic Target. Intensive Care Med. 46 (4), 586-590. doi:10.1007/s00134-020-05985-9 
Zhang, H., Zahid, A., Ismail, H., Tang, Y., Jin, T., and Tao, J. (2020b). An Overview of Disease Models for NLRP3 Inflammasome Over-activation. Expert Opin. Drug Discov. 16, 429-446. doi:10.1080/17460441.2021. 1844179

Zhang, L.-L., Huang, S., Ma, X.-X., Zhang, W.-Y., Wang, D., Jin, S.-Y., et al. (2016). Angiotensin(1-7) Attenuated Angiotensin II-Induced Hepatocyte EMT by Inhibiting NOX-Derived H 2 O 2 -activated NLRP3 inflammasome/IL-1 $\beta$ / Smad Circuit. Free Radic. Biol. Med. 97, 531-543. doi:10.1016/j.freeradbiomed. 2016.07.014

Zhao, C., and Zhao, W. (2020). NLRP3 Inflammasome-A Key Player in Antiviral Responses. Front. Immunol. 11, 211. doi:10.3389/fimmu.2020. 00211

Zhao, M., Bai, M., Ding, G., Zhang, Y., Huang, S., Jia, Z., et al. (2018). Angiotensin II Stimulates the NLRP3 Inflammasome to Induce Podocyte Injury and Mitochondrial Dysfunction. Kidney Dis. 4 (2), 83-94. doi:10.1159/000488242
Zhou, P., Yang, X.-L., Wang, X.-G., Hu, B., Zhang, L., Zhang, W., et al. (2020). A Pneumonia Outbreak Associated with a New Coronavirus of Probable Bat Origin. Nature 579 (7798), 270-273. doi:10.1038/s41586020-2012-7

Conflict of Interest: The authors declare that the research was conducted in the absence of any commercial or financial relationships that could be construed as a potential conflict of interest.

Copyright $(2021$ Zhang, Tang and Tao. This is an open-access article distributed under the terms of the Creative Commons Attribution License (CC BY). The use, distribution or reproduction in other forums is permitted, provided the original author(s) and the copyright owner(s) are credited and that the original publication in this journal is cited, in accordance with accepted academic practice. No use, distribution or reproduction is permitted which does not comply with these terms. 